\title{
Hydrogen Sensor Based on Palladium-Yttrium Alloy Nanosheet
}

\author{
Boyi Wang ${ }^{\mathrm{a}}$, Yong Zhu ${ }^{\mathrm{a}}$, Youping Chen ${ }^{\mathrm{b}}$, Han Song ${ }^{\mathrm{b}}$, Pengcheng Huang ${ }^{\mathrm{b}}$, Dzung Viet Dao ${ }^{\mathrm{a}}$ \\ ${ }^{a}$ Queensland Micro- and Nanotechnology Centre, Griffith University, Nathan, QLD 4111, Australia \\ ${ }^{\mathrm{b}}$ School of Mechanical Science and Engineering, Huazhong University of Science and Technology, Wuhan 430074, \\ China
}

\begin{abstract}
This paper presents a hydrogen sensor based on palladium-yttrium (Pd-Y) alloy nanosheet. Zigzag-shaped Pd-Y nanosheet with a thickness of $19.3 \mathrm{~nm}$ was deposited on a quartz substrate by using an ultrahigh-vacuum magnetron sputtering system and shadow mask. The ratio of palladium to yttrium in the nanosheet was $0.92 / 0.08$. The fabrication process was simple and low-cost, and the sensor can be mass-produced. The experimental results show the sensor has a superior sensitivity, reversibility, and reproducibility. The resistive-based hydrogen detection mechanism in this research is much simpler and more compact compared to the optical-based detection method.
\end{abstract}

\section{Highlights}

- $\quad$ Pd-Y sensing element was fabricated using a magnetron sputtering system and shadow mask.

- $\quad$ The Pd-Y compound consisted of $92 \% \mathrm{Pd}$ and $8 \%$ Y.

- The fabrication process was simple, low-cost, and mass-production compatible.

- The sensor showed superior sensitivity, reversibility, and reproducibility to hydrogen gas.

- $\quad$ The device is more compact than the optical-based counterpart.

Keywords: Nanosheet, magnetron sputtering system, Pd-Y alloy, shadow mask, resistive-base hydrogen sensor

\section{Introduction}

Hydrogen gas is colourless, odourless, tasteless, and highly flammable. There has been a great demand for fast response and highly sensitive hydrogen gas sensors for safety and industrial application to alert the formation of potentially explosive gas and to reduce the explosion risk [1]. Presence detection and concentration measurement of hydrogen gas have over 100 years of history $[1,2]$. However, higher sensitivity and faster response hydrogen sensors are desirable for further safety improvement.

Hydrogen sensing element is normally made by metal or alloy, whose physical and chemical properties vary when it is exposed to hydrogen gas. A good sensing material should have high selectivity, sensitivity, and reversibility to hydrogen. Compared to other materials, such as platinum, palladium was found to have higher solubility and sensitivity to hydrogen [1, 3, 4]. The absorption of hydrogen molecule by pure palladium can form $\mathrm{Pd}-\mathrm{H}$ hydride, leading to the changes of its physical properties, such as mass, volume, optical property, and electrical resistivity [5]. During the desorption process, the palladium thin film operates under non-equilibrium conditions. The concentration of hydrogen in the thin film is higher than that in the surface film. Due to the concentration gradient, the chemical bonds between palladium and hydrogen atoms in the thin film are broken and the hydrogen atoms move to the surface of film and are desorbed to the atmosphere. In order to gain high activation energy to break $\mathrm{Pd}-\mathrm{H}$ bonds, the concentration gradient between the thin film and the exposed environment should be sufficiently high enough to provide the necessary driving force [14]. However, pure palladium film is easy to bubble and crack after absorbing and desorbing hydrogen for several times [6], reducing the stability and reliability of the sensor. Mechanical stress in the film and lattice expansion of palladium structure are the significant issues for further research to address [7].

In literature, there are mainly two solutions that are able to avoid the crack of pure palladium thin film after absorbing and desorbing hydrogen. The first approach is to expand the lattice of Pd before the invasion of hydrogen atoms. The expanded lattice leaves larger space for hydrogen atoms to diffuse in $\mathrm{Pd}$ thin film. As a result, the $\alpha$ to $\beta$ phase transition in $\mathrm{PdH}_{\mathrm{x}}$ is suppressed when $\mathrm{Pd}$ absorbs hydrogen. Liu et al. [8] found that Palladiumyttrium (Y) alloy thin film could improve the response rate and eliminate the bubble and delamination phenomena. As yttrium atom has larger radius than palladium atom, the lattice of palladium will expand after being doped by yttrium. The expanded lattice makes the diffusion of hydrogen atoms in Pd-Y alloy thin film much easier. The main advantage of his research was simple fabrication process by using DC/RF magnetron sputtering system. The deposition rates of two metals were monitored by quartz crystal thickness monitor. The surface morphology of thin film in the SEM was clean and flat.

The second approach to address the fracture issue of pure palladium film is to utilize low-dimensional structures, such as nanowire and nanoparticle [10-13]. Pd film tends to expand due to its volume increase when it absorbs hydrogen gas, leading to a compressive stress at the interface of the film and substrate [14]. Conversion of materials into nanoscale reduces crystal defects, thereby enhancing mechanical strength and reducing the fracture phenomenon. Furthermore, nanostructures have been a major research focus as high performance hydrogen sensing elements. Since nanostructures have a large surface to volume ratios, the hydrogen diffusion paths can be shortened, and absorption and desorption rates of chemical reaction are improved. Palladium nanostructures have shown promising properties, which are suitable for high speed, high sensitivity, miniature size, and low-cost 
hydrogen sensor [13]. However, the nanowire and nanoparticle are of great complexity to fabricate and they normally require expensive nano-fabrication facilities for mass-production.

Pd-Y alloy thin film has been used to detect hydrogen gas based on both optical and electrical methods [6-9, 15]. The electrical detection method is inexpensive, easy and convenient compared to the optical counterpart [6-9]. Furthermore, palladium-yttrium based gas sensor does not require high operating temperature, which is compulsory in other popular resistive gas sensors based on tungsten oxide $[16,17]$

Jamshidi et al. [15] reported the hydrogen detection based on the resistance change of palladium-yttrium thin film. However, the resistance change was small due to its large thickness and unpatterned sensing element. Moreover, the results were not precise and reliable due to long time intervals of data collection.

Wang et al. [5] did research on zigzag-shaped palladium-silver thin film for hydrogen sensing based on electronic resistance change. The zigzag-shaped thin film can provide larger electrical resistance and change than unpatterned thin film. Therefore, the sensor showed higher sensitivity and reversibility. However, complex fabrication processes were required to pattern the thin film.

In this paper, we present a novel zigzag-shaped resistive hydrogen sensor based on $\mathrm{Pd}_{0.92}-\mathrm{Y}_{0.08}$ alloy nanosheet. The sensing element has an ultra-thin thickness of $19.3 \mathrm{~nm}$ and reasonable electrical resistance of $7 \mathrm{k} \Omega$. The fabrication process of the resistive hydrogen sensor is simple and has fewer steps by utilizing shadow mask and ultrahigh-vacuum magnetron sputtering system. The proposed fabrication process is suitable for massproduction of the sensor.

\section{Experiment}

\subsection{Sensor fabrication}

Fig. 1 illustrates the fabrication process of the hydrogen sensor based on patterned Pd-Y alloy nanosheet. The substrate is a round-shaped quartz glass with $20 \mathrm{~mm}$ in diameter and $3 \mathrm{~mm}$ in thickness. First, the quartz glass substrate and shadow mask were cleaned in ethanol using ultrasonic cleaner. The shadow mask is required for patterning the alloy nanosheet to increase the nominal resistance of the sensing element. To fabricate the shadow mask, a thin plastic sheet was cut into zigzag-shaped using laser cutter (TROTEC Speedy $300^{\mathrm{TM}}$ ). The shadow mask was attached to the surface of glass substrate, as shown in Fig.1 (a).
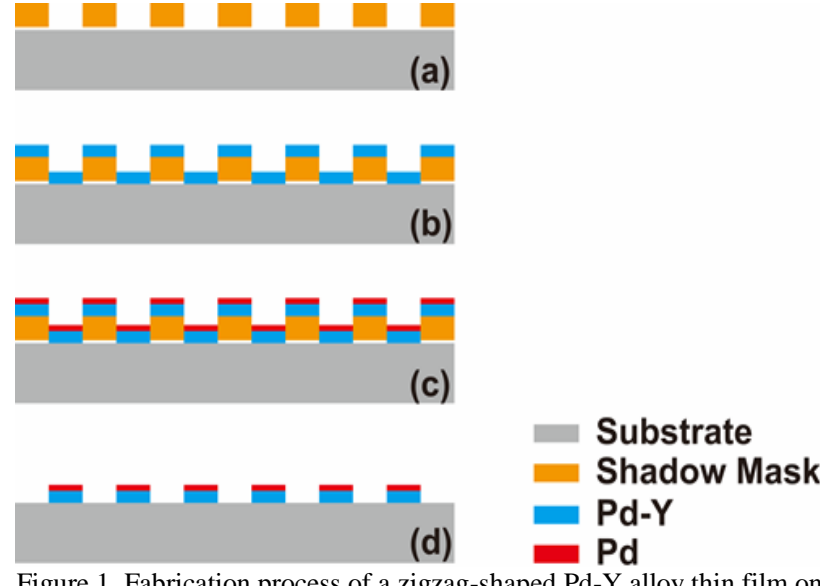

Figure 1. Fabrication process of a zigzag-shaped Pd-Y alloy thin film on quartz glass substrate.

To deposit the ultra-thin film of palladium-yttrium alloy, ultrahigh vacuum magnetron sputtering system (BESTEC) was utilized. Palladium and yttrium targets with purity of $99.95 \%$ were installed in the DC and RF sources of the sputtering system, respectively. The thickness of the alloy nanosheet was monitored by quartz crystal thickness monitor with a resolution of $0.1 \mathrm{~nm}$. The detailed process parameters of magnetron sputtering process are listed in Table 1 . A $15 \mathrm{~nm}$ Pd-Y nanosheet was deposited on the quartz glass substrate using BESTEC sputtering system. The Pd-Y alloy nanosheet was patterned by the zigzag-shaped shadow mask as shown in Fig. 1 (b).

Table 1. Process parameters of magnetron sputtering process.

\begin{tabular}{lll}
\hline \multicolumn{1}{c}{ Parameters } & DC & RF \\
Target material & $\mathrm{Pd}$ & $\mathrm{Y}$ \\
Working gas & $\mathrm{Ar}$ & $\mathrm{Ar}$ \\
Working pressure (mbar) & $5 \times 10^{-3}$ & $5 \times 10^{-3}$ \\
Sputtering power $(\mathrm{W})$ & 130 & 30 \\
Deposition rate $(\AA / \mathrm{s})$ & 1.0 & 0.1 \\
\hline \hline
\end{tabular}

However, yttrium is very easy to be oxidized when Pd$\mathrm{Y}$ material is exposed to air. To avoid this problem, a thin protection film is required on the top of the Pd-Y alloy nanosheet. The pure palladium can be a good candidate because it does not react with oxygen. Therefore, the DC sputtering source continued to operate for 50 seconds after the RF sputtering was shut down. A thin palladium layer is coated on the surface of Pd-Y alloy nanosheet, and the thickness of the layer is $5 \mathrm{~nm}$ as illustrated in Fig. 1 (c). The pure ultra-thin palladium layer can allow hydrogen atoms to penetrate into the Pd-Y alloy to react, meanwhile it can reduce the probability of the contact between yttrium atoms in the alloy and oxygen atoms in air [7, 8]. At last, the zigzag-shaped nanosheet was remained on the quartz glass substrate after removal of the shadow mask as shown in Fig. 1 (d).

Fig. 2 depicts a schematic diagram of the zigzagshaped resistive hydrogen sensor. The sensing material was deposited in the middle of substrate. The material of both electrodes was same as sensing element with size of $2 \mathrm{~mm} \times 2 \mathrm{~mm}$. All the measurement and discussion hereafter are based on designs with a width of $500 \mu \mathrm{m}$, length of $92 \mathrm{~mm}$ for the zigzag-shaped nanosheet. 


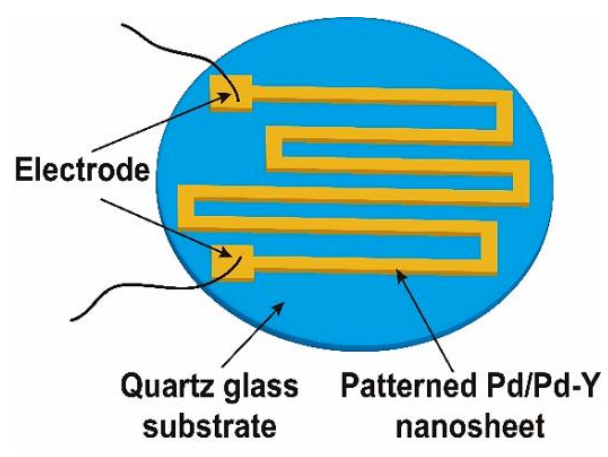

Figure 2. Schematic of the hydrogen sensor.

\subsection{Characterization of the Pd-Y alloy nanosheet}

The thickness of the patterned alloy nanosheet was measured to be $19.3 \mathrm{~nm}$ using profilometer (KLATENCOR P16). This is the total thickness of $\mathrm{Pd}_{0.92}-\mathrm{Y}_{0.08}$ layer and pure Pd layer. The surface characterization of the nanosheet was analysed by Atomic Force Microscopy (AFM), as plotted in Fig. 3. The Pd-Y alloy nanosheet was scanned and analysed by AFM, and the roughness of this alloy nanosheet was $0.827 \mathrm{~nm}$. The horizontal and vertical scanning resolutions were $0.26 \mathrm{~nm}$ and $1 \mathrm{~nm}$, respectively.

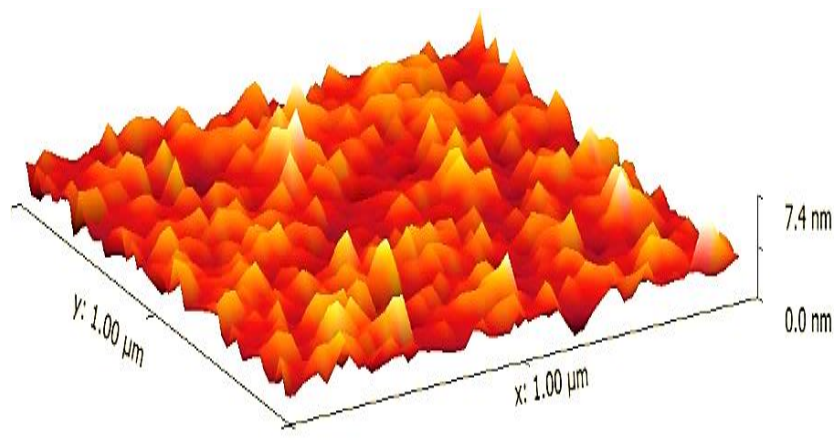

Figure 3. AFM image of Pd-Y alloy nanosheet surface nanocrystalline profile.

\subsection{Hydrogen sensor testing system}

The resistive hydrogen sensor was tested in a $5 \mathrm{~cm}$ cube gas chamber, which was designed for the electrical hydrogen detection system. The hydrogen sensor was located in the center of the gas chamber. The sensor electrodes were adhered with two $\mathrm{Cu}$ wires by silver epoxy conductive adhesive. The curing time for the contact points were approximately 24 hours at room temperature or 30 minutes at $100^{\circ} \mathrm{C}$. The contact point became hard with high electrical conductivity after curing. As shown in Fig. 4, the hydrogen gas sources with various concentrations of $0.05 \%, 0.5 \%$, and $4 \%$ were connected to the gas chamber via rubber tubing and controlled by three valves. The hydrogen gas is introduced continuously into the gas chamber with a set flow rate of $200-300 \mathrm{ml} / \mathrm{min}$ in standard condition. The valve of hydrogen source was turned off after each test, and the gas chamber was opened to remove the remaining hydrogen gas naturally. A PC connected with a precision LCR meter (HPS2816A) was utilized for all measurement data collection and analysis. The change of resistance with hydrogen was recorded by precision LCR meter with time intervals of 1 second. The gas response $\left(G_{\mathrm{s}}\right)$ is defined as the percentage of resistance change when the sensor is exposed to the hydrogen gas as follows:

$$
\mathrm{G}_{\mathrm{S}}(\%)=\frac{\mathrm{R}_{\mathrm{h}}-\mathrm{R}_{\mathrm{a}}}{\mathrm{R}_{\mathrm{a}}} \times 100 \%=\frac{\Delta \mathrm{R}}{\mathrm{R}_{\mathrm{a}}} \times 100 \%
$$

where $R_{a}$ and $R_{h}$ are the resistances of hydrogen sensor device before and after its exposure to a specific hydrogen concentration, respectively. In this research, the range of hydrogen concentrations was set from $0.05 \%$ to $4 \%$ at the room temperature and pressure.

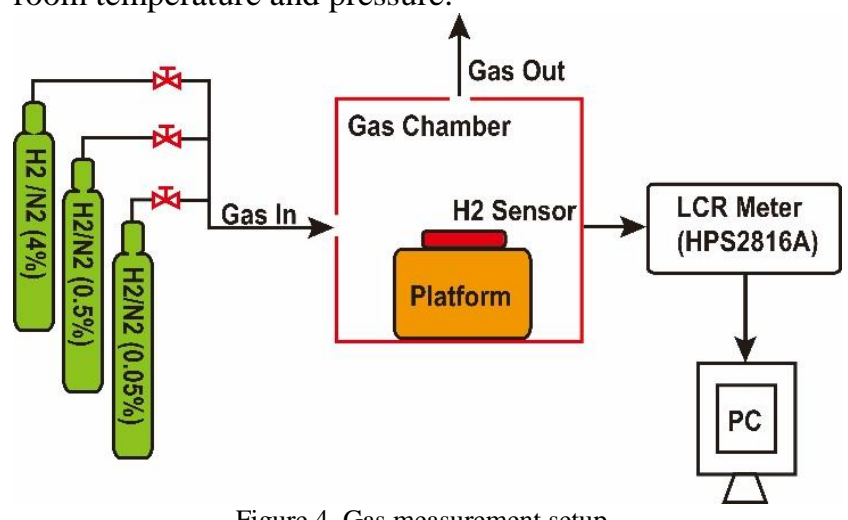

Figure 4. Gas measurement setup.

\section{Results and discussion}

Fig. 5 shows the response of $\mathrm{Pd}-\mathrm{Y}$ based hydrogen sensor at various hydrogen concentrations. Multi-cycle measurement of the resistive hydrogen sensor was carried out with exposure to $4 \%, 0.5 \%$, and $0.05 \%$ concentrations of hydrogen gas. Each concentration of hydrogen gas was turned on and off for a few cycles, as shown in Fig. 5. Due to safety consideration, the concentration of hydrogen gas in this research was limited to $4 \%$. The gas response was increased very abruptly when $4 \%$ hydrogen gas was introduced into gas chamber, and it rapidly decreased after gas source was switched off. However, in the case of lower hydrogen concentration of $0.05 \%$, the gas response was slower and more placid due to less hydrogen molecules on the nanosheet surface for reaction. The results in Fig. 5 indicate that the novel resistive hydrogen sensor has good stability and repeatability when concentrations of hydrogen changed. In addition, the nominal resistance of the hydrogen sensor was measured to be around $7 \mathrm{k} \Omega$, which is large enough to facilitate the resistance measurement and improve the signal to noise ratio for the sensor.

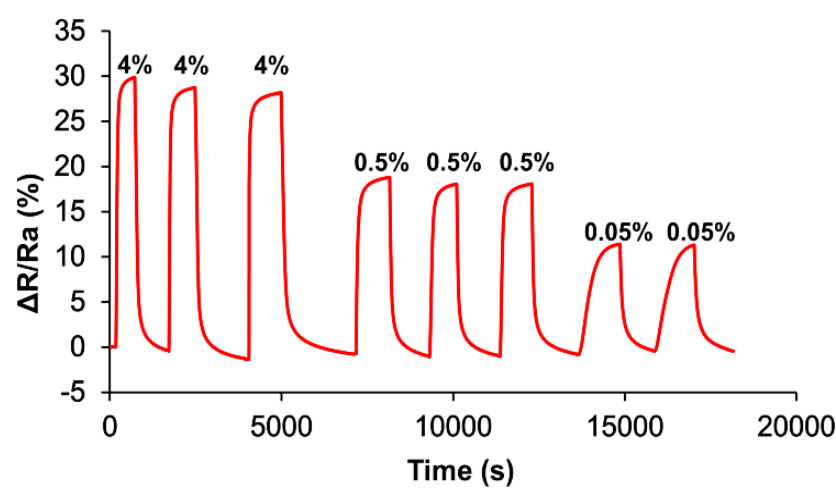

Figure 5. Relative resistance changes of the Pd-Y nanosheet versus hydrogen gas concentrations $(4 \%, 0.5 \%, 0.05 \%$ in nitrogen). 
The response time is defined as the required time from initial point to $90 \%$ of the stable point, and the recovery time is defined as the required time from stable value to $10 \%$ of the stable value when hydrogen gas stops to charge into gas chamber. As shown in Fig. 6, the response and recovery times at $0.05 \%$ hydrogen gas concentration were 630 seconds and 390 seconds, respectively. The response time and recovery time decreased sharply to 83 seconds and 210 seconds, respectively, when hydrogen concentration changed from $0.05 \%$ to $4 \%$. When the nanosheet is exposed to hydrogen gas, dissociation of hydrogen molecules on the surface and diffusion in the PdY structure could affect the sensor response time [14]. Therefore, the response could be faster by reducing the thickness of Pd-Y nanosheet. Moreover, the recovery time could be reduced by using nitrogen gas or vacuum pump to remove remaining hydrogen gas in gas chamber.

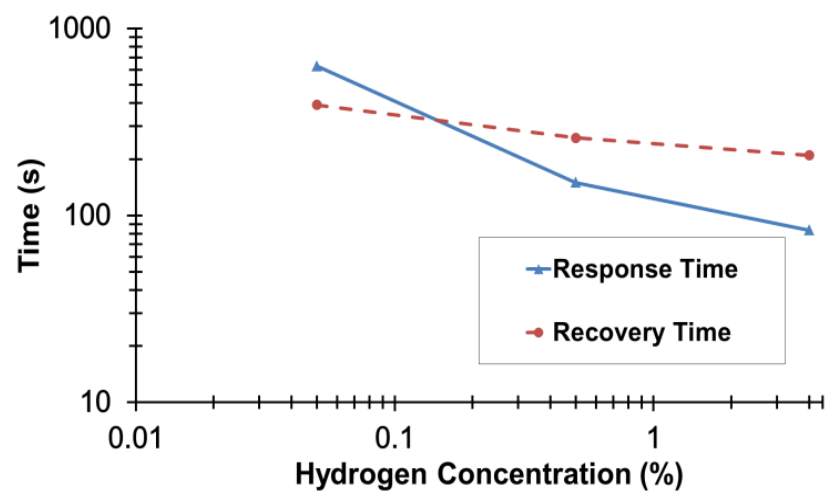

Figure 6. Response and recovery times of the Pd-Y nanosheet sensor versus hydrogen concentrations.

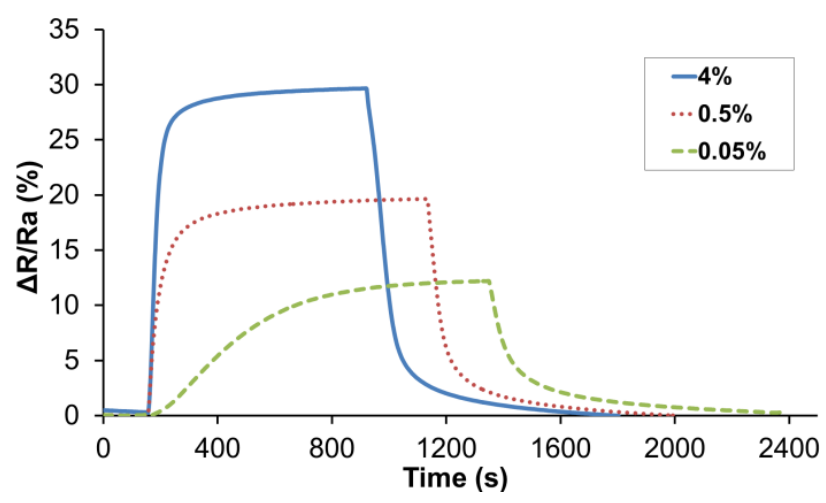

Figure 7. Response curves of the sensor for different hydrogen concentrations.

Fig. 7 shows normalized gas response for $0.05 \%, 0.5 \%$ and $4 \%$ hydrogen concentrations at room temperature. After exposing the resistive hydrogen sensor to $4 \%$ hydrogen, the gas response of the hydrogen sensor increased rapidly to $29.28 \%$ within approximate one and half minutes. After discharging hydrogen, the resistance of the hydrogen sensor decreased sharply to the initial value within around 100 seconds, as shown in Fig. 7. Three different hydrogen concentration curves in Fig. 7 were redrawn to start at the same time. More hydrogen atoms on nanosheet surface lead to faster $\alpha$ to $\beta$ transition, resulting in shorter response and recovery times [3].

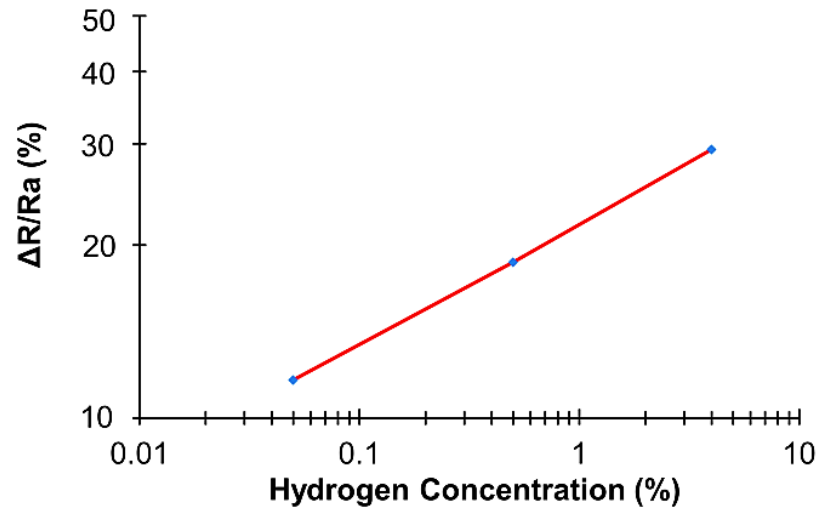

Figure 8. Relationship between the sensor response and the specific hydrogen concentration.

The gas response of the Pd-Y based hydrogen sensor versus hydrogen concentration is shown in Fig. 8. The hydrogen concentrations in this research were $0.05 \%$, $0.5 \%$, and $4 \%$, and the corresponding gas response were $11.64 \%, 18.67 \%$ and $29.28 \%$, respectively. The relation between hydrogen concentration and gas response was found to be linear and plotted in Fig. 8. The sensitivity of this novel resistive hydrogen sensor is improved due to the large gas response $\left(\Delta R / R_{a}\right)$. The sensitivity of this sensor is based on material property change in $\mathrm{Pd}-\mathrm{Y}$ alloy nanosheet due to the invasion of hydrogen atoms and the $\alpha$ to $\beta$ transition. The measured gas response of this hydrogen sensor was larger than the previous work reported in [5, 18, 19]. A detailed comparison with other works is shown in Table 2.

Table 2. Comparison with other works.

\begin{tabular}{|c|c|c|c|c|}
\hline & $\begin{array}{c}\text { Wang et al. } \\
{[5]}\end{array}$ & $\begin{array}{c}\text { Kim et al. } \\
{[18]}\end{array}$ & $\begin{array}{c}\text { Jeon et al. } \\
{[19]}\end{array}$ & This work \\
\hline Fabrication & $\begin{array}{c}\text { Complex } \\
\text { Costly }\end{array}$ & $\begin{array}{c}\text { Complex } \\
\text { Costly }\end{array}$ & $\begin{array}{c}\text { Complex } \\
\text { Costly }\end{array}$ & $\begin{array}{c}\text { Simple } \\
\text { Low-cost }\end{array}$ \\
\hline Thickness & $200 \mathrm{~nm}$ & $50 \mathrm{~nm}$ & $20-400 \mathrm{~nm}$ & $19.3 \mathrm{~nm}$ \\
\hline Structure & $\begin{array}{c}\text { Zigzag- } \\
\text { shaped film }\end{array}$ & $\begin{array}{c}\text { Net-shaped } \\
\text { film }\end{array}$ & Nanowire & $\begin{array}{c}\text { Zigzag-shaped } \\
\text { nanosheet }\end{array}$ \\
\hline Material & $\begin{array}{c}\text { Palladium- } \\
\text { Silver }\end{array}$ & $\begin{array}{c}\text { Palladium } \\
\text { Ralladium }\end{array}$ & $\begin{array}{c}\text { Palladium- } \\
\text { Yttrium }\end{array}$ \\
\hline Response & $\begin{array}{c}15 \mathrm{~s} \\
(2 \%)\end{array}$ & $\begin{array}{c}42 \mathrm{~s} \\
(0.2 \%)\end{array}$ & $\begin{array}{c}7 \mathrm{~s} \\
(1 \%, 20 \mathrm{~nm})\end{array}$ & $\begin{array}{c}83 \mathrm{~s} \\
(4 \%)\end{array}$ \\
\hline $\begin{array}{c}\text { Time } \\
\text { Response }\end{array}$ & $\begin{array}{c}15 \% \\
(2 \%)\end{array}$ & $\begin{array}{c}0.783 \% \\
(0.2 \%)\end{array}$ & $\begin{array}{c}3.83 \% \\
(1 \%, 20 \mathrm{~nm})\end{array}$ & $\begin{array}{c}18.67 \% \\
(0.5 \%)\end{array}$ \\
\hline
\end{tabular}

\section{Conclusions}

A zigzag-shaped resistive hydrogen sensor based on Pd-Y alloy nanosheet was fabricated on a quartz glass substrate with a simple nanofabrication technology using an ultrahigh-vacuum magnetron sputtering system and shadow mask. The Pd-Y alloy nanosheet was characterized by AFM and profilometer. The actual thickness of the nanosheet was $19.3 \mathrm{~nm}$. The palladium and yttrium in the nanosheet was controlled as $92 \%$ and $8 \%$, respectively. The novel resistive hydrogen sensor showed high sensitivity, superior reversibility, and reproducibility. The resistive-based detection method is 
much convenient and easier compared to optical detection counterpart. The fabrication of this novel hydrogen sensor is simple, inexpensive and fast. Therefore, the sensor can be mass-produced. The patterned Pd-Y nanosheet with nano-metre thickness and unique alloy material offers a superior gas response at room temperature and pressure.

\section{Acknowledgments}

This work is financially supported by Science and Technology department of Hubei province (number: 2016AHB019). The authors would like to thank Wuhan National Laboratory for Optoelectronic and Huazhong University of Science and Technology for providing micofabrication and characterization facilities.

\section{References}

[1] T. Hübert, L. Boon-Brett, G. Black, U. Banach, Hydrogen sensors a review, Sens.Actuators B: Chem. 157 (2011) 329-352.

[2] MAN Company, Vorrichtung zur fortlaufenden Bestimmung des Wasserstoffgehaltes in Gasgemischen, Patent DRP 165349 (1904).

[3] S. H. Lim, B. Radha, J. Y. Chan, Mohammad S. M. Saifullah, Giridhar U. Kulkarni, G.W Ho, Flexible palladium-based H2 sensor with fast response and low leakage detection by nanoimprint lithography, ACS Appl. Mater. Interfaces. 5 (2013) 7274-7281.

[4] F.A. Lewis, The Palladium-Hydrogen System, Academic Press, 1967.

[5] M. Wang, Y, Feng, palladium-silver thin film for hydrogen sensing, Sens and Actuators B: Chem. 123 (2007) 101-106.

[6] Y. Liu, Y. P. Chen, H. Song, G. Zhang, Modelling analysis and experimental study on the optical fiber hydrogen sensor based on Pd-Y alloy thin film, Rev. Sci.Instrum. 83 (2012) 075001-075005.

[7] H. Song, Y. P. Chen, G. Zhang, Y. Liu, P. C. Huang, H. W. Zhao, M. H. Yang, J. X. Dai, Z. Li, Optical fiber hydrogen sensor based on an annealing-stimulated Pd-Y thin film, Sensors and Actuators B Chemical. 216 (2015) 11-16.

[8] Y. Liu, Y. P. Chen, H. Song, G. Zhang, Hydrogen gas sensor based on palladium and yttrium alloy ultrathin film, Rev. Sci. Instrum. 83 (2012) 125003-125008.

[9] Y. Liu, Y. Chen, H. Song, G. Zhang, Characteristics of an optical fiber hydrogen gas sensor based on a palladium and yttrium alloy thin film, Sens. J. IEEE 13(2013) 2699-2704

[10] Y. J. Lim, Y. J. Lee, J. Heo, H. J. Shin, Highly sensitive hydrogen gas sensor based on a suspended palladium/carbon nanowire fabricated via batch microfabrication process. Sensors and Actuators B: Chemical. 210 (2015) 218-224.

[11] F. Yang, D.K. Taggart, R.M. Penner, Fast, sensitive hydrogen gas detection using single palladium nanowires that resist fracture Nano Lett. 9 (2009) 2177-2182.

[12] X. Q. Zeng, M. L. Latimer, Z. L. Xiao, S. Panuganti, U. Welp, W. K. Kwok, T. Xu, Hydrogen gas sensing with networks of ultrasmall palladium nanowires formed on filtration membranes, Nano Lett. 11 (2011) 262-268

[13] X. Q. Zeng, Y. L. Wang, H. Deng, M. L. Latimer, Z. L. Xiao, J. Pearson, T. Xu, H. H. Wang, U. Welp, G. W. Crabtree, W. K. Kwok, Networks of ultrasmall $\mathrm{Pd} / \mathrm{Cr}$ nanowires as high performance hydrogen sensors, ACS Nano. 5 (2011) 7443-7452.

[14] E. Lee, J. M. Lee, J. H. Koo, W. Lee, T. Lee, Hysteresis behaviour of electrical resistance on $\mathrm{Pd}$ thin films during the process of absorption and desorption of hydrogen gas, International Journal of Hydrogen Energy 35 (2010) 6984-6991.

[15] M M. Jamshidi, K. Alshaltami, F. Akkari, J. Wright, Development of palladium-based hydrogen thin film sensor using silicon oxide substrate, Indian J Phys. 87 (2013) 511-515.

[16] J. Tamaki, T. Hashishin, Y. Uno, Dzung V. Dao, S. Sugiyama, "Ultrahigh-sensitive WO3 Nanosensor with Interdigitated Au Nano-electrode for NO2 Detection", Sensors and Actuators B, Vol.132, pp. 234-238, 2008.

[17] Dzung V Dao, K Shibuya, Tung $\mathrm{T}$ Bui and S Sugiyama, "Micromachined NH3 Gas Sensor with ppb-level Sensitivity Based on WO3 Nanoparticles Thin film”, Procedia Engineering, Vol.25, 2011, pp. 1149-1152
[18] B.J. Kim, J.S. Kim, Hydrogen sensor using the Pd film supported on anodic aluminium oxide, International Journal of Hydrogen Energy 39 (2014) 16500-16505.

[19] K.J. Jeon, J.M. Lee, E. Lee and W. Lee, Individual Pd nanowire hydrogen sensors fabricated by electron-beam lithography. Nanotechnology 20 (2009) 1355021-1355025. 